IPMU-10-0103

NSF-KITP-10-085

\title{
Black holes in an asymptotically safe gravity theory with higher derivatives
}

\author{
Yi-Fu Cai ${ }^{1,2,3, *}$ and Damien A. Easson ${ }^{2,3,4, \dagger}$ \\ 1 Institute of High Energy Physics, Chinese Academy of Sciences, \\ P.O. Box 918-4, Beijing 100049, China \\ ${ }^{2}$ Department of Physics \& School of Earth and Space Exploration \& Beyond Center, \\ Arizona State University, Tempe, AZ 85287, USA \\ 3 Institute for the Physics and Mathematics of the Universe, \\ University of Tokyo, 5-1-5 Kashiwanoha, Kashiwa, Chiba 277-8568, Japan \\ ${ }^{4}$ Kavli Institute for Theoretical Physics, \\ University of California, Santa Barbara, CA 93106-4030, USA
}

We present a class of spherically symmetric vacuum solutions to an asymptotically safe theory of gravity containing high-derivative terms. We find quantum corrected Schwarzschild-(anti)-de Sitter solutions with running gravitational coupling parameters. The evolution of the couplings is determined by their corresponding renormalization group flow equations. These black holes exhibit properties of a classical Schwarzschild solution at large length scales. At the center, the metric factor remains smooth but the curvature singularity, while softened by the quantum corrections, persists. The solutions have an outer event horizon and an inner Cauchy horizon which equate when the physical mass decreases to a critical value. Super-extremal solutions with masses below the critical value correspond to naked singularities. The Hawking temperature of the black hole vanishes when the physical mass reaches the critical value. Hence, the black holes in the asymptotically safe gravitational theory never completely evaporate. For appropriate values of the parameters such stable black hole remnants make excellent dark matter candidates.

\footnotetext{
*Email:caiyf@ihep.ac.cn

${ }^{\dagger}$ Email:easson@asu.edu
} 


\section{INTRODUCTION}

One of the most challenging tasks facing theoretical physicists today is the construction of a consistent ultraviolet (UV) complete theory of gravity. Weinberg has suggested that the effective quantum field description of a gravitation theory may be UV complete and nonperturbatively renormalizable by virtue of asymptotic safety (AS) [1]. In this scenario the renormalization group (RG) flows have a fixed point in the UV limit and a finite dimensional critical surface of trajectories approach this point at short distances. This theory has been extensively studied in the literature [2-9], and recent evidence suggests the UV critical surface is only three-dimensional even in truncations of the exact RG equations with more than three independent coupling parameters [10-15]. Until now, the majority of the work on the subject has considered significant truncations of the action, taking into account only the Einstein-Hilbert and occasionally cosmological constant terms. In this paper, we initiate the study of black hole solutions in an asymptotically safe gravity theory including higher derivative terms and running gravitational couplings. While we focus on the above goal, our methodology is easily generalized to include a broader analysis of black solutions in higher derivative theories.

Our concrete starting point is a generally covariant gravitational theory with effective action involving a momentum cutoff $p$ :

$$
\begin{aligned}
\Gamma_{p}\left[g_{\mu \nu}\right]= & \int d^{4} x \sqrt{-g}\left[p^{4} g_{0}(p)+p^{2} g_{1}(p) R\right. \\
& +g_{2 a}(p) R^{2}+g_{2 b}(p) R_{\mu \nu} R^{\mu \nu} \\
& \left.+g_{2 c}(p) R_{\mu \nu \sigma \rho} R^{\mu \nu \sigma \rho}+\mathcal{O}\left(p^{-2} R^{3}\right)+\cdots\right]
\end{aligned}
$$

where $g$ is the determinant of the metric tensor $g_{\mu \nu}, R$ is the Ricci scalar, $R_{\mu \nu}$ is the Ricci tensor and $R_{\mu \nu \lambda \sigma}$ is the Riemann tensor. The coefficients $g_{i}(i=0,1,2 a, \ldots)$ are dimensionless coupling parameters and are functions of the dimension-full, UV cutoff. In particular, for long wavelengths we have

$$
g_{0}(p)=-\frac{\Lambda(p)}{8 \pi G_{N}(p)} p^{-4}, \quad g_{1}(p)=\frac{1}{8 \pi G_{N}(p)} p^{-2},
$$

where it is useful to define the dimensionless Newton's constant $\bar{G}_{N}(p)=G_{N}(p) p^{2}$ and dimensionless cosmological constant $\bar{\Lambda}(p)=\Lambda(p) p^{-2}$. The couplings satisfy the following 
RG equations,

$$
\frac{d}{d \ln p} g_{i}(p)=\beta_{i}[g(p)]
$$

The conditions for asymptotic safety require that all the beta functions vanish $\beta_{i}=0$ when the coupling parameters $g_{i}$ approach a fixed point $g_{i}^{*}$. If $g_{i}^{*}=0$, the fixed point is Gaussian; if $g_{i}^{*} \neq 0$, the fixed point is Non-Gaussian (NG). For the NG fixed point, all the coupling parameters are fixed, the cutoff $p$ becomes irrelevant as $p \rightarrow \infty$, and the theory is adequately described by a finite number of higher order counter-terms included in the effective action. Near the fixed point we may Taylor expand the beta functions in a matrix form

$$
\beta_{i}[g]=\sum_{j} \mathcal{B}_{i j}\left(g_{j}-g_{j}^{*}\right)
$$

where the elements of the matrix are defined by $\mathcal{B}_{i j} \equiv{\frac{\partial \beta_{i}\left[g_{j}\right.}{\partial g_{j}}}^{*}$ at the fixed point. Solving the RG equations (3) in the neighborhood of the fixed point we find

$$
g_{i}(p)=g_{i}^{*}+\sum_{m} e_{i}^{n}\left(\frac{p}{M_{*}}\right)^{v_{n}},
$$

where $e^{n}$ and $v_{n}$ are the suitably normalized eigenvectors and corresponding eigenvalues of the matrix $\mathcal{B}_{i j}$. Since $\mathcal{B}$ is a general real matrix with symmetry determined by a particular gravity model, its eigenvalues can be either real or in pairs of complex conjugates. As a consequence, the dimensionality of the ultraviolet critical surface is equal to the number of eigenvalues of the matrix $\mathcal{B}$, of which the real parts take negative values. The above solution involves an arbitrary mass scale $M_{*}$. By requiring the largest eigenvector of order unity, $M_{*}$ is typically identified with the energy scale at which the coupling parameters are just beginning to approach the fixed point.

It is well known that the quantization procedure for General Relativity leads to a nonrenormalizable quantum field theory, where an infinite number of terms have to be fixed to renormalize the standard perturbation theory. Including higher derivative terms in the action (such as we have done in (1)) introduces higher derivative propagators that soften the divergences of the perturbative quantization and can result in a perturbatively renormalizable theory [16]. Unfortunately, the higher derivative terms required, introduce new massive spin-two degrees of freedom with wrong-sign kinetic term (i.e. negative norm states, 
or ghosts) [17]. For example, if we quantize this system in a canonical form the problem with ghosts arises since the metric field and its high-derivative term are regarded as two independent variables. These instabilities generically render the theory non-unitary and unstable. However, as shown in [18], if the quantum theory is based on a path integral which is evaluated in Euclidean space and then Wick rotated to Lorentzian space, the path integral can yield a theory of quantum gravity without a negative norm state. A specific application of this approach in inflationary cosmology provides an interesting interpretation for metric perturbations [19]. Surprizingly, evidence is mounting that these catastrophic ghosts may be eliminated in higher derivative theories when the UV limit of gravity is controlled by a NG fixed point as in the AS gravity scenario [12, 20-23].

Because the asymptotically safe gravity theory is relevant for the physics of high energy scales and short distances, one is naturally lead to consider its application to early universe cosmology. For example, Weinberg has recently argued for the existence of inflationary solutions complete with graceful exit in the context of the theory [15]. Another clear application of AS gravity is to black hole physics; in particular, to understand how the theory modifies the conventional Schwarzschild black hole solution by taking into account the quantum corrections naturally incorporated into the model. Some early attempts are presented in [24-27]. Their key assumption was that the leading order quantum corrections to the black hole spacetime are captured via a simple running of the Newtonian constant $G_{N}$ determined by the renormalisation group equation for gravity; however, the consistency of the resultant modified metric and modified effective action was not thoroughly investigated. For simplicity, the analysis was limited to a severe truncation of the action (1), including only the $g_{1}$ term. By truncating to the Einstein-Hilbert term (as in any $f(R)$ truncation) the authors eliminated the four-derivative propagator for the helicity two states which may drastically alter the AS theory and its solutions. In this paper, we develope an effective method of finding vacuum solutions to Einstein's equation derived from the AS gravity with higher derivative terms. We focus on spherically symmetric spacetimes and present an exact form of a Schwarzschild-(anti)-de-Sitter (SAdS) solution. The thermodynamical properties of these black hole solutions are briefly discussed.

The paper is organized as follows: in $\S I I$, we derive the equation of motion for the AS high-derivative gravity and study a generic vacuum solution to this theory which preserves static spherical symmetry. We find that, to leading order, this solution is exactly SAdS. 
Due to the quantum nature of renormalization, the coupling coefficients vary with respect to the running of energy scale and it is necessary to investigate the RG flow of these coupling coefficients in accordance with the asymptotically safe scenario (see, §III). In $\S I V$, we analyze the black hole solutions to the theory. We determine the scale identification between the momentum cutoff and the radial coordinate and subsequently, we study the behavior of the background geometry in the UV and infrared (IR) limits. We find that the metric factor is nonsingular for all values of the radial coordinate but the curvature diverges at the origin. Moreover, there exists a critical mass for the background geometry. When the black hole mass is heavier than the critical value the black hole has two horizons, but this solution vanishes when the mass is subcritical, revealing a naked singularity. Numerical calculations are performed to confirm our analytic results. The black hole thermodynamics are briefly discussed and we present our concluding remarks in $\S \mathrm{V}$.

\section{STATIC SPHERICALLY SYMMETRIC VACUUM SOLUTIONS}

Black hole physics provides a window into the quantum nature of gravity. Black holes possess many remarkable properties, for example, the associated thermodynamics [28] and holographic properties. The simplest black hole solution in four dimensions corresponds to a Schwarzschild spacetime $[29,30]$. As is well known, the geometry is divided into two causally independent regions by an event horizon located at the Schwarzschild radius $r_{s}=2 G_{N} M^{1}$. Given the possibility of an asymptotically safe gravitational theory, it is logical to re-examine these intriguing properties of black hole physics when quantum corrections are incorporated into the gravitational action. We now obtain the field equations from the action (1) and search for static, spherically symmetric solutions to the theory.

The generalized Einstein field equations are obtained by varying the action (1) with respect to the metric tensor $g_{\mu \nu}$ :

$$
\tilde{G}^{\mu \nu} \equiv \frac{\delta \Gamma_{p}\left[g_{\mu \nu}\right]}{\delta g_{\mu \nu}}=T^{\mu \nu}
$$

where $\tilde{G}^{\mu \nu}$ is the generalized Einstein tensor and $T^{\mu \nu}$ is the energy-momentum tensor of the background matter and is vanishing due to our vacuum ansatz. The generalized Bianchi

\footnotetext{
${ }^{1}$ Here and throughout we use Natural units with $c=\hbar=k_{B}=1$.
} 
identity is preserved automatically due to the general covariance of the scalar-type action. The form of $\tilde{G}^{\mu \nu}$ appearing in the above equation can be expressed as,

$$
\begin{aligned}
\tilde{G}^{\mu \nu}= & \frac{1}{2} g^{\mu \nu}\left(p^{4} g_{0}+p^{2} g_{1} R+g_{2 a} R^{2}+g_{2 b} R_{\sigma \rho} R^{\sigma \rho}+g_{2 c} R_{\sigma \rho \lambda \kappa} R^{\sigma \rho \lambda \kappa}\right)-p^{2} g_{1} R^{\mu \nu} \\
& +g_{2 a}\left(-2 R R^{\mu \nu}+\nabla^{\mu} \nabla^{\nu} R+\nabla^{\nu} \nabla^{\mu} R-2 g^{\mu \nu} \square R\right) \\
& +g_{2 b}\left(-2 R_{\rho}^{\mu} R^{\nu \rho}+\nabla_{\rho} \nabla^{\mu} R^{\rho \nu}+\nabla_{\rho} \nabla^{\nu} R^{\rho \mu}-\square R^{\mu \nu}-g^{\mu \nu} \nabla^{\rho} \nabla^{\sigma} R_{\rho \sigma}\right) \\
& +g_{2 c}\left(-2 R^{\mu \rho \sigma \lambda} R_{\rho \sigma \lambda}^{\nu}-2 \nabla_{\rho} \nabla_{\sigma} R^{\mu \rho \nu \sigma}-2 \nabla_{\rho} \nabla_{\sigma} R^{\nu \rho \mu \sigma}\right) .
\end{aligned}
$$

In the above, we have defined the d'Alembertian operator $\square \equiv \nabla_{\mu} \nabla^{\mu}$, where $\nabla_{\mu}$ is the covariant derivative. The trace of the Einstein tensor gives the useful quantity,

$$
\begin{aligned}
\tilde{G} & \equiv g_{\mu \nu} \tilde{G}^{\mu \nu} \\
& =2 p^{4} g_{0}+p^{2} g_{1} R-2\left(3 g_{2 a}+g_{2 b}+g_{2 c}\right) \square R,
\end{aligned}
$$

which vanishes in our vacuum investigation, $T^{\mu \nu}=0$. In the above derivation we have applied the Bianchi identity: $\nabla_{\sigma}\left(R^{\sigma \rho}-\frac{1}{2} g^{\sigma \rho} R\right)=0$.

We assume a static, spherically symmetric metric ansatz,

$$
d s^{2}=-A(r) d t^{2}+\frac{d r^{2}}{B(r)}+r^{2} d \Omega_{2}^{2} .
$$

Substituting the metric (9) into the action (1) and neglecting the time integral, up to order $\mathcal{O}\left(p^{0}\right)$ gives,

$$
\begin{aligned}
\Gamma_{p}[A, B]= & \int d r 4 \pi r^{2} \sqrt{\frac{A}{B}}\left(g_{0} p^{4}+g_{1} p^{2} R+g_{2 a} R^{2}\right. \\
& \left.+g_{2 b} R_{\mu \nu} R^{\mu \nu}+g_{2 c} R_{\mu \nu \rho \sigma} R^{\mu \nu \rho \sigma}\right)
\end{aligned}
$$

where the evaluated curvature invariants in terms of the metric (9) are included in the Appendix. To obtain the vacuum solutions we must solve:

$$
\frac{\delta \Gamma_{p}}{\delta A}=\frac{\delta \Gamma_{p}}{\delta B}=0
$$

The above calculation is greatly simplified by choosing the Schwarzschild gauge $B(r)=$ $N(r) A(r)$ and setting $N=1$. This relation is ensured by the Cauchy theorem for a Riemannian geometry with unique boundary. 
Varying the action with respect to the function $A(r)$ gives:

$$
\begin{aligned}
\frac{\delta \Gamma}{\delta A}= & \frac{4 \pi}{r^{2}}\left[4\left(3 g_{2 a}+g_{2 b}+g_{2 c}\right)\left(-2+2 A-r^{2} A^{\prime \prime}\right)\right. \\
& \left.+\left(2 g_{2 a}+g_{2 b}+2 g_{2 c}\right) r^{3}\left(4 A^{(3)}+r A^{(4)}\right)\right] \\
= & 0 .
\end{aligned}
$$

Solving the above equations of motion yields,

$$
A(r)=1+c_{1} r^{n_{1}}+c_{2} r^{n_{2}}+\frac{c_{3}}{r}+c_{4} r^{2},
$$

where the exponents $n_{j}(j=1,2)$ are given in terms of the couplings $g_{2 k}(k=a, b, c)$,

$$
n_{1(2)}=\frac{1}{2}\left(1 \mp \sqrt{\frac{50 g_{2 a}+17 g_{2 b}+18 g_{2 c}}{2 g_{2 a}+g_{2 b}+2 g_{2 c}}}\right) .
$$

This solution involves four coefficients $c_{1}, c_{2}, c_{3}$ and $c_{4}$ which must be determined by other constraint equations, boundary conditions and the consistency relation with infrared limit solution.

To determine the coefficients appearing in the solution (13), we impose the constraint equation $\tilde{G}=0$, consistent with our vacuum assumption. A natural choice is to set $c_{1}=$ $c_{2}=0$, giving the SAdS solution to the high-derivative gravity theory:

$$
A(r)=1-\frac{2 G_{p} M}{r}-\frac{r^{2}}{l_{p}^{2}}
$$

where $G_{p}$ is a gravitational coupling which is varying along with the running of the cutoff scale. The parameter $M$ is an integration constant we identify with the physical mass of the black hole, and $l_{p}$ is the radius of the asymptotic (A)dS space which is also a cutoff dependent function, expressed as

$$
l_{p}^{2} \simeq-\frac{6 g_{1}}{g_{0} p^{2}}\left[1+\sqrt{1-\frac{g_{0}}{3 g_{1}^{2}}\left(12 g_{2 a}+3 g_{2 b}+2 g_{2 c}\right)}\right] .
$$

The solution is consistent with the usual vacuum solution from GR when the energy scale flows to the IR limit, but differs significantly at high energy scales, as we shall see. We require a scale identification between the coefficients of the solution and the cutoff scale $p$ which is dependent on the details of the AS gravity theory. Therefore, in the subsequent section we apply the AS scenario of the high-derivative gravity action. 


\section{ASYMPTOTICALLY SAFE HIGH-DERIVATIVE GRAVITY}

The functional gravitational RG equation is based on a momentum cutoff for the propagating degrees of freedom and captures the nonperturbative information about the gravitational theory. The RG equation is of the form [4]:

$$
\frac{\partial}{\partial \ln p} \Gamma_{p}=\frac{1}{2} \operatorname{Tr}\left(\delta^{(2)} \Gamma_{p}+\mathbf{R}(p)\right) \frac{\partial}{\partial \ln p} \mathbf{R}(p),
$$

where $\mathbf{R}$ is an appropriately defined momentum cutoff at the scale $p$ and is usually determined by the so-called optimized cutoff process $[31,32]$. In the above formula, we suppose the gauge fixing terms have already been included, although they are irrelevant for our present consideration. Additionally, the trace in the RG equation stands for a sum over spacetime indices and a loop integration. Our philosophy then is to effectively integrate out the high momentum fluctuations with momentum larger than the cutoff $p$, and incorporate them via the modified dynamics for the fluctuations having momentum less than $p$.

The high-derivative terms in the effective action (1) can be regrouped as follows,

$$
\Gamma_{p}^{\mathrm{HD}}=\int d^{4} x \sqrt{-g}\left[\frac{\omega}{3 \lambda} R^{2}-\frac{1}{2 \lambda} C^{2}+\frac{\theta}{\lambda} E\right],
$$

where

$$
C^{2} \equiv C_{\mu \nu \rho \sigma} C^{\mu \nu \rho \sigma}=R_{\mu \nu \rho \sigma} R^{\mu \nu \rho \sigma}-2 R_{\mu \nu} R^{\mu \nu}+\frac{R^{2}}{3}
$$

is the square of the 4-dimensional Weyl tensor, and

$$
E=R_{\mu \nu \rho \sigma} R^{\mu \nu \rho \sigma}-4 R_{\mu \nu} R^{\mu \nu}+R^{2}
$$

is the integrand of the Gauss-Bonnet term which is topological in 4-dimensional spacetime. The re-expressed high-derivative terms in Eq. (17) are equivalent to the terms appearing in the original action (1), under the following identifications,

$$
\begin{aligned}
& g_{2 a}=-\frac{1}{6 \lambda}+\frac{\theta}{\lambda}+\frac{\omega}{3 \lambda} \\
& g_{2 b}=\frac{1}{\lambda}-\frac{4 \theta}{\lambda} \\
& g_{2 c}=-\frac{1}{2 \lambda}+\frac{\theta}{\lambda} .
\end{aligned}
$$

Note that a standard derivation of dimensional regularization with $d=4-\epsilon[33,34]$ indicates that the beta functions of the dimensionless coefficients introduced in (1) are 
sensitive to the dimensional corrections for the case, $\epsilon<0$. Therefore, we restrict our attention to the case of $\epsilon \geq 0$. Explicitly in the nontrivial limit of $\epsilon \rightarrow 0$, the beta functions for the dimensionless coefficients of the high-derivative gravitational terms are given by [33$35]$,

$$
\begin{aligned}
& \beta_{\lambda}=-\frac{1}{(4 \pi)^{2}} \frac{133}{10} \lambda^{2}, \\
& \beta_{\omega}=-\frac{1}{(4 \pi)^{2}}\left(\frac{5}{12} \lambda+\frac{183}{10} \lambda \omega+\frac{10}{3} \omega^{2}\right), \\
& \beta_{\theta}=-\frac{1}{(4 \pi)^{2}}\left(-\frac{196}{45} \lambda+\frac{133}{10} \lambda \theta\right) .
\end{aligned}
$$

To perform the stability analysis, we note that the coefficient $\lambda$ has the familiar logarithmic form which approaches asymptotic freedom,

$$
\lambda(p)=\frac{\lambda_{0}}{1+\frac{133}{(4 \pi)^{2} 10} \lambda_{0} \ln p / M_{p}},
$$

where $\lambda_{0}$ is a fixed value of the coefficient $\lambda$ at the Planck scale. Since the above form is logarithmic, we find $\lambda \simeq \lambda_{0}$ in a wide range around the Planck scale. We will apply this approximation in deriving the coordinate dependent cutoff scale later. Moreover, the other two parameters $\theta$ and $\omega$, approach a group of fixed points in the UV limit, among which the stable ones take the values

$$
\theta^{*} \simeq 0.327, \quad \omega^{*} \simeq-0.0228,
$$

where the superscript " $*$ " denotes the parameter value at the NG fixed point as introduced in the beginning of this paper.

By solving the beta functions for the gravitational coupling and cosmological constant, we observe a Gaussian fixed point in the IR limit and a NG fixed point in the UV limit. The central result is

$$
\begin{aligned}
& g_{0} \simeq-\frac{\left(\Lambda_{I R}+\eta p^{2} G_{N}\right)\left(1+\xi p^{2} G_{N}\right)}{8 \pi p^{4} G_{N}}, \\
& g_{1} \simeq \frac{1+\xi p^{2} G_{N}}{16 \pi p^{2} G_{N}},
\end{aligned}
$$

where $G_{N}$ and $\Lambda_{I R}$ are the values of the gravitational coupling and the cosmological constant in the IR limit which are determined by astronomical observations. In the UV limit, the coefficients of the Einstein-Hilbert part of the gravity action will flow to a NG fixed point 
with $g_{0} \rightarrow g_{0}^{*}$ and $g_{1} \rightarrow g_{1}^{*}$. To obtain the NG fixed point values of the remaining variables in the high-derivative terms we solve the flow equation explicitly

$$
\begin{aligned}
& g_{0}^{*} \simeq-\frac{\eta \xi G_{N}}{8 \pi} \simeq-6.331 \times 10^{-3}, \\
& g_{1}^{*} \simeq \frac{\xi}{16 \pi} \simeq 1.432 \times 10^{-2},
\end{aligned}
$$

by a numerical computation. Notice that the running gravitational coupling $G_{p}$ is related to the coefficient $g_{1}$ by,

$$
G_{p}=\frac{1}{16 \pi p^{2} g_{1}}=\frac{G_{N}}{1+\xi p^{2} G_{N}} .
$$

From the analysis of $g_{1}$, we see that $G_{p}$ coincides with the Newtonian constant $G_{N}$ at low energy scales but decreases rapidly as the momentum cutoff $p$, which implies a weakening of gravity at high energy scales. We will see that this weakening of the gravitational force in the UV leads to a softening of the singular behavior of the black hole solution near the origin.

\section{BLACK HOLES \& ASYMPTOTICALLY SAFE GRAVITY}

From the above analysis, we find that the spherically symmetric vacuum solutions to the high-derivative gravity theory flow to the classical SAdS geometry at low energy scales. However, in order to implement quantum corrections to the running coefficients appearing in the classical geometry, we must determine the relationship between the momentum cutoff $p$ and the radial coordinate $r$.

\section{A. Relevant scale}

Recall the quantity $\tilde{G}$, introduced in Eq. (7), vanishes for the vacuum state, $T_{\mu \nu}=0$. Inserting the solution (14) into Eq. (7) and making use of the redefinition (20), we could, in principle, solve for the momentum cutoff $p$ as a function of the radial coordinate $r$ by requiring $\tilde{G}=0$, which gives

$$
\tilde{G}=2 p^{4} g_{0}+p^{2} g_{1} R-\frac{2 \omega}{\lambda} \square R=0 .
$$

Note that the above equation is a fourth-order differential equation and will yield a class of solutions for the momentum cutoff $p(r)$. Consequently, it is necessary to impose the relevant 
constraints in order to determine the physical solution. We now focus our attention to the black hole solutions at high energy scales and at small radial distances compared with the Planck scale, $l_{p}$.

As a consequence of requiring $\tilde{G}=0$, we derive a scale identification between the momentum scale $p$ and the coordinate $r$ of the form,

$$
p(r) \simeq 2.663\left(\frac{M^{2}}{\left|\lambda_{0}\right|}\right)^{\frac{1}{8}} r^{-\frac{3}{4}}
$$

when the energy scale is as high as the AS scale. We note that this relation is different from the result $p \sim r^{-3 / 2}$ appearing in [27] through a UV matching. The discrepancy is due to the action truncation considered in [27], which consisted of only the EinsteinHilbert term and a vanishing cosmological constant. In the present analysis, we include the higher derivative terms and nonzero cosmological constant in addition to the EH term and running $G_{N}$. Consequently, we consider the effective action in the low energy limit. When the momentum cutoff flows to the IR regime, the high-derivative terms are suppressed automatically and thus becomes negligible. In this case, $p \sim 1 / r$ which is consistent with the IR matching of [27]. The asymptotically safe SAdS geometry is obtained by replacing the classical gravitational constant $G_{N}$ and the $(\mathrm{A}) \mathrm{dS}$ radius with a $\mathrm{RG}$ improved $G_{p}(r)$ and $l_{p}(r)$, respectively.

Note that the $\mathrm{dS}$ radius $l_{p}$ has significant cosmological implications and may be related to the nearly exponential acceleration of our universe at early times, as well as, the cosmological acceleration occurring today. The running of $l_{p}$ can drive an early inflationary period, complete with a successful graceful exit [15]. For late time cosmological acceleration, we may consider the possibility that the value of $l_{p}$ in the IR limit is of order of the size of our universe in accordance with the latest cosmological observations. Such cosmological applications are beyond the scope of the present analysis.

\section{B. Black hole solutions}

Let us focus our interests on the local quantities of the quantum corrected black hole solutions (especially near the horizon and the origin) and leave the investigation of dS radius to future study. One can finely tune a very small value of $\lambda_{0}$ in order to let the dS radius be much larger than the black hole horizon. In the following we study this geometry 
along with the momentum cutoff $p$ flowing from the UV regime to the IR regime.

\section{UV limit}

As the radial coordinate approaches the origin, the energy scale of the cutoff could be very high, and consequently the coupling parameters may already have arrived at their NG fixed point. Correspondingly, the gravitational coupling is expressed as

$$
G_{p}(r)=\frac{1}{16 \pi p^{2}(r) g_{1}} \simeq \frac{1}{\xi p^{2}(r)}
$$

when $p \gg M_{p}$. In this case, the coefficient $\lambda$ approaches zero, leading to infinitely large $g_{2}$. According to Eq. (15), the radius of the asymptotical dS space diverges. Therefore, we can neglect the last term of the solution (14) near the origin. Inserting Eqs. (32) and (33) into (14), we derive the approximate form of the metric factor in the UV limit:

$$
A_{U V}(r) \simeq 1-\frac{625}{512 \pi}\left|\lambda_{0}\right|^{\frac{1}{4}}(M r)^{\frac{1}{2}} .
$$

The above result indicates that the metric factor $A(r)$, unlike its GR counterpart, is no longer singular inside the horizon of the black hole in AS gravity with leading order highderivative terms. Naively, it may appear that the geometry is glued to an asymptotical Minkowski spacetime near the origin. However, substituting the solution into the expression for the Ricci scalar, we obtain

$$
R \simeq \frac{9375}{2048 \pi} \frac{M^{\frac{1}{2}}\left|\lambda_{0}\right|^{\frac{1}{4}}}{r^{\frac{3}{2}}},
$$

which is singular at the origin of the spacetime. By applying the horizon condition $A_{U V}(r)=$ 0, we obtain an approximate solution

$$
r_{U V} \simeq \frac{0.671 \pi^{2}}{\left|\lambda_{0}\right|^{\frac{1}{2}} M}
$$

at high energy scales, indicating that the curvature singularity is hidden behind the UV horizon. The other familiar invariants are altered as well and reflect the softening of the singular behavior in the asymptotically safe gravity theory:

$$
\begin{aligned}
R_{\mu \nu \lambda \sigma} R^{\mu \nu \lambda \sigma} & \simeq \frac{31640625}{4194304} \frac{\sqrt{\lambda_{0}}}{\pi^{2}} \frac{M}{r^{3}} \\
C_{\mu \nu \lambda \sigma} C^{\mu \nu \lambda \sigma} & \simeq \frac{1171875}{4194304} \frac{\sqrt{\lambda_{0}}}{\pi^{2}} \frac{M}{r^{3}} \\
R_{\mu \nu} R^{\mu \nu} & \simeq \frac{59765625}{8388608} \frac{\sqrt{\lambda_{0}}}{\pi^{2}} \frac{M}{r^{3}} .
\end{aligned}
$$


These should be compared with their hard singularity partners in General Relativity, for example, the Kretschmann scalar (37) in GR diverges at the origin as $\sim 1 / r^{6}$.

\section{IR limit}

Along with the increasing of the radial coordinate the momentum cutoff could drop to the regime below the Planck scale as $p \sim 1 / r$. In this case,

$$
G_{p}(r) \simeq G_{N}\left(1-\frac{\tilde{\xi} G_{N}}{r^{2}}\right)
$$

where $\tilde{\xi}$ is a constant which deviates from the coefficient $\xi$ by a factor of $\mathcal{O}(1)$. Substituting this gravitational coupling parameter into the black hole solution yields

$$
A_{I R}(r) \simeq 1-\frac{2 G_{N} M}{r}\left(1-\frac{\tilde{\xi} G_{N}}{r^{2}}\right) .
$$

By solving the equation of horizon condition, we obtain another real solution which corresponds to the event horizon at low energy scales. Expanding to the leading order in the coefficient $\tilde{\xi}$, we obtain the familiar Schwarzschild horizon value,

$$
r_{I R} \simeq 2 G_{N} M-\frac{\tilde{\xi}}{2 M}+\mathcal{O}\left(\tilde{\xi}^{2}\right) .
$$

Hence, the horizon location of the AS black hole is in precise agreement with the usual form for the Schwarzschild radius $r_{s}=2 G_{N} M$ in the limit $\tilde{\xi} \rightarrow 0$.

By comparing the solution for the event horizon in the UV limit (36) and the horizon in the IR limit (42), we see there is a critical value for the mass parameter of the black hole:

$$
M_{c} \sim\left|\lambda_{0}\right|^{-\frac{1}{4}} G_{N}^{-\frac{1}{2}}
$$

When $M>M_{c}$, the black hole in AS high-derivative gravity has two horizons. There is an inner Cauchy horizon as well as an outer event horizon corresponding to that of the ordinary black hole solution in GR. At the extremal value $M=M_{c}$, these two horizons coincide; beyond the extremal value $\left(M<M_{c}\right)$ the black hole vanishes, corresponding to a naked singularity. The double horizon feature of our solution is in agreement with the results obtained in the AS gravity with Einstein truncation analyzed in [24].

Here we make further comparison between our results and those obtained in Refs. [24-27]. First, due to the presence of higher derivative terms, the form of the scale identification (32) 
is altered compared with the result obtained in the case of the pure Einstein truncation [27]. Furthermore, the black hole solutions obtained with Einstein truncation are smooth and nonsingular, but become singular if higher derivative terms are taken into account. The conditions identifying the critical mass are the same in the two scenarios, i.e., both the metric factor and its derivative with respect to the mass are vanishing.

\section{Numerical analysis}

In the above analytic study we made a number of approximations in order to obtain the black hole solutions in the AS high-derivative gravity theory. To better understand the analytic results, we now turn to a quantitative numerical analysis. Our results for the coordinate-dependent momentum cutoff $p(r)$ and the quantum corrected Schwarzschild metric factor $A(r)$ are presented in Figs. 1 and 2, respectively.

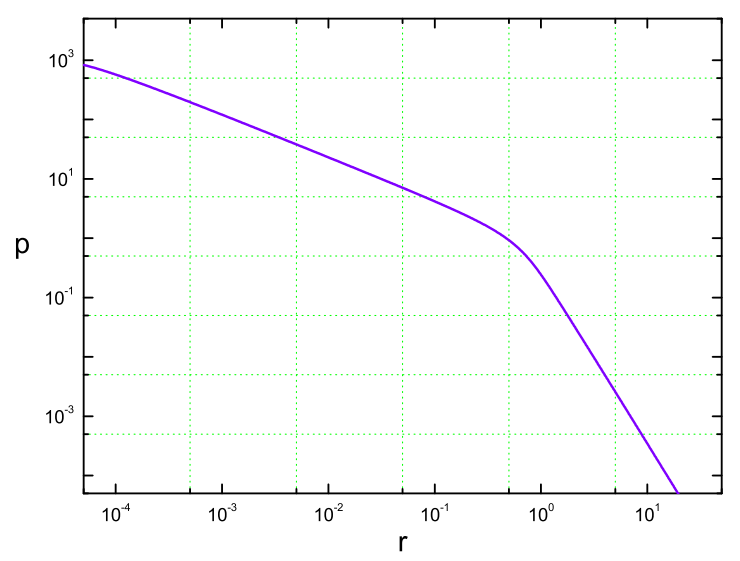

FIG. 1: Running of the momentum cutoff $p$ as a function of the radial coordinate $r$. Here we take $\lambda_{0}=G_{N}=1$.

Fig. 1, shows the slope of the running momentum cutoff curve in the IR and UV regimes. In the IR regime, which corresponds to a large length scale, $p$ scales approximately as $1 / r$. However, in the high energy limit, the relation between the momentum cutoff and the radial coordinate becomes $p \propto r^{-3 / 4}$ which is in agreement with the analytic result obtained in Eq. (32).

In Fig. 2, we plot the $g_{00}$ metric component of the spherically symmetric vacuum solutions 


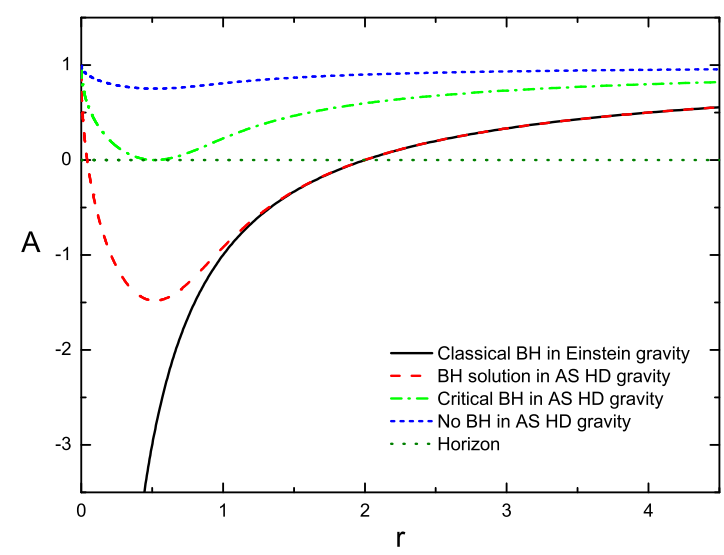

FIG. 2: The metric factors $A$ of the spherically symmetric vacuum solutions as functions of the radial coordinate $r$ in both Einstein gravity and the AS high-derivative gravity. In the numerical computation, we take $\lambda_{0}=G_{N}=1$. We consider $M=1, M=0.403$ and $M=0.1$, which correspond to a RG running black hole, a critical black hole, and a naked singularity, respectively.

of AS high-derivative gravity for the values of the mass parameter: $M=1, M=0.403$ and $M=0.1$. In order to illustrate the significance of the quantum corrections to the vacuum solutions, we compare them with the metric factor of a black hole in classical Einstein gravity as denoted by the black solid curve. The red dash curve corresponds to the metric factor of a double-horizon black hole with $M=1$ in AS high-derivative gravity. When the physical mass decreases to the critical value $\left(M_{c}=0.403\right.$ in this example), we obtain an extremal black hole and the outer horizon coincides with the inner horizon. This case is plotted by the green dash-dot curve. Moreover, the blue short dash curve shows that there are no black hole solutions (i.e. there is a naked singularity) for subcritical values of the mass $M<M_{c}$. In all of the above solutions the metric factors of the background geometries are smooth and nonsingular, but the curvature is divergent at the origin.

\section{Thermodynamics of the IR horizon}

From the above analysis, we have shown how the normal Schwarzschild geometry is corrected by nonperturbative effects in quantum gravity. To further probe the quantum gravity effects we now study the process of black hole evaporation via Hawking radiation [28]. 
We apply the Euclidean path integral approach to determine the temperature and the specific heat [36], and employ the method of complex path analysis to study the emission rate of the Hawking radiation [37, 38]. We now investigate the thermodynamical features of the quantum corrected black hole around the IR horizon in the super-critical solution with $M \geq M_{c}$

There has been considerable interest in the study of thermodynamics of quantum corrected black holes. For example, the Hawking radiation of a two dimensional nonsingular black hole [39] in the context of dilaton gravity [40,41]. Similar to the derivation performed in [39], we compute the Bekenstein-Hawking temperature

$$
\begin{aligned}
T & =\frac{1}{4 \pi} A^{\prime}\left(r_{I R}\right) \\
& \simeq \frac{1}{8 \pi G_{N} M}\left(1-\frac{\tilde{\xi}}{4 G_{N} M^{2}}\right),
\end{aligned}
$$

around the IR horizon. In comparison with the standard result, the temperature of the quantum corrected black hole is smaller than that of the classical analog (see Fig. 3). Stefan's

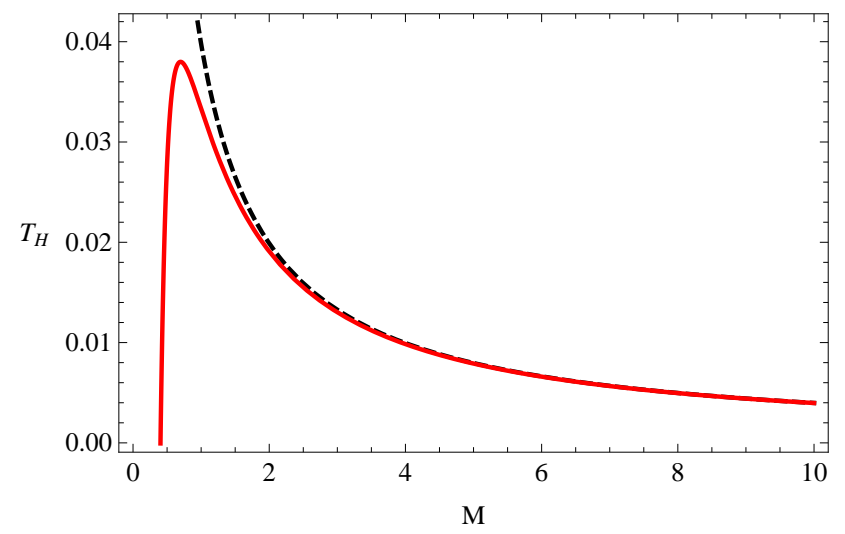

FIG. 3: Approximate Hawking temperature as a function of black hole mass (see Eq. (44)). The AS high-derivative solution is the solid (red) curve and is compared with the standard Hawking temperature of General Relativity $T^{-1}=8 \pi M$, dashed (black) curve, which diverges for small $M$. In the numerical computation, we take $\tilde{\xi}=.65$ and $G_{N}=1$. The temperature reaches a maximum at $M=\sqrt{3 \tilde{\xi} / 4 G_{N}}$ corresponding to $T_{\max }^{-1}=6 \pi \sqrt{3 G_{N} \tilde{\xi}}$, and reaches zero at the critical value $M_{c}=\sqrt{\tilde{\xi} / 4 G_{N}} \simeq .403$. 
law gives the black hole radiation power law derivation:

$$
\begin{aligned}
\mathcal{P} & =-\frac{d M}{d t}=4 \pi \sigma r_{I R}^{2} T^{4} \\
& \simeq \frac{\sigma}{256 \pi^{3} G_{N}^{2} M^{2}}\left(1-\frac{3 \tilde{\xi}}{2 G_{N} M}\right)
\end{aligned}
$$

where $\sigma=\pi^{2} / 60$ is a Stefan-Boltzmann constant. Hence, the evaporation of the quantum corrected black hole can only take place when its physical mass is heavier than the critical value. Consider $\lambda_{0} \sim \mathcal{O}(1)$, this critical value coincides with the critical mass $M_{c}$ as introduced in the previous subsection. Once the physical mass is smaller than $M_{c}$, the absorption process will dominate over the emission process of the black hole radiation. Therefore, a critical mass black hole with $M \sim M_{c}$ is expected to be the most stable. Indeed, from Fig. (3) we see that the extremal black hole ceases to decay because the temperature vanishes for the critical mass.

From the radiation power law obtained above, it is possible to estimate the evaporation time of the black hole taking into account the quantum gravity corrections

$$
\tau \sim \frac{M}{|\mathcal{P}|} \sim \frac{\tau_{S}}{1-\frac{3 \tilde{\xi}}{2 G_{N} M}},
$$

where $\tau_{S}$ is the evaporation time of a classical black hole. The quantum corrected evaporation time is infinite when the physical mass of the black hole decreases to the scale around the critical mass $M_{c}$, in accordance with our expectations.

In the above analysis we studied the black hole thermodynamics in the framework of the AS high-derivative gravity theory using perturbative methods with $\tilde{\xi}<1$. However, we do not expect these qualitative results to be drastically altered in the nonperturbative regime. This is due to the fact that there is always a critical mass for the black hole solution where its IR horizon coincides with the UV horizon. This corresponds to an extremal black hole with a vanishing temperature (since $T \propto A^{\prime}=0$ ). As a consequence, the black hole evaporation time will be infinite as discussed above.

\section{CONCLUSIONS}

In this paper we have initiated the study of static spherically symmetric vacuum solutions to the theory of asymptotically safe gravity with high-derivative corrections. We find that 
a generic solution corresponds to a quantum corrected SAdS spacetime due to the RG flows of the gravitational coupling parameters. Under certain simplifying assumptions, we obtain a black hole solution with smooth metric factor and a curvature singularity at the center of the geometry. The singular behavior is mildly softened in the AS high derivative theory compared to the corresponding GR black hole. The quantum gravity corrected black hole solution generically possesses two horizons, which correspond when the physical mass of the black hole decreases to a critical value. The temperature of the black hole is exactly zero when its physical mass reaches the critical value. We find that the temperature of the quantum corrected black hole is, in general, lower than that of a classical black hole and stable critical mass remnants are natural final states of the Hawking evaporation process.

These results are related to many other interesting issues which deserve future study. For example, a collection of critical black holes may serve as an alternative candidate for dark matter if their masses are as low as the TeV scale. From Eq. (43), we find that this is a possibility if $\lambda_{0} \sim 10^{64}$ which is also compatible with the theoretical requirement of suppressing gravitational high-derivative terms. The passive thermodynamic properties of the AS black hole with a critical mass may leave significant signals on the matter power spectrum at small length scales. In addition, if sufficient numbers of critical mass holes were produced at the end of inflation, they may leave potentially measurable signals in the polarization of the cosmic microwave background radiation on small angular scales. A potential concern is that if the critical mass of a black hole is much lower than the Planck scale Minkowski spacetime could become unstable due to the perturbation modes which increase the mass of the black hole. It is then possible that there would be too many primordial black hole remnants produced in a static background. We note that this problem may be alleviated in realistic cosmological models due to the expansion of the universe. As the expansion of the background spacetime dominates over the production rate of black holes, the density of primordial black holes may be significantly diluted after a sufficiently long period. We leave the study of this intriguing possibility for future work.

\section{Acknowledgments}

It is a pleasure to thank R. Brandenberger, R. Emparan, K. Stelle and S. Weinberg for helpful conversations. YFC acknowledges Prof. Xinmin Zhang for extensive support of his 
research. YFC thanks the Institute for the Physics and Mathematics of the Universe and the Research Center for the Early Universe at the University of Tokyo, Tokyo University of Science, and the Yukawa Institute for Theoretical Physics at Kyoto University for their hospitality when this work was finalized. The work of YFC is supported in part by the National Science Foundation of China under Grants No. 10533010 and 10803001. DAE would like to thank the Yukawa Institute for Theoretical Physics at Kyoto University for their hospitality during YKIS2010 (YITP-T-10-01) where this work was completed. The work of DAE is supported in part by the World Premier International Research Center Initiative (WPI Initiative), MEXT, Japan and by a Grant-in-Aid for Scientific Research (21740167) from the Japan Society for Promotion of Science (JSPS), and by funds from the Arizona State University Foundation, and by the National Science Foundation under Grant No. PHY05-51164.

[1] S. Weinberg, Critical Phenomena for Field Theorists, in Understanding the Fundamental Constituents of Matter, ed. A. Zichichi (Plenum Press, New York, 1977).

[2] in General Relativity, ed. S. W. Hawking and W. Israel (Cambridge University Press, 1979): 790.

[3] H. Kawai, Y. Kitazawa and M. Ninomiya, "Ultraviolet stable fixed point and scaling relations in (2+epsilon)-dimensional quantum gravity," Nucl. Phys. B 404, 684 (1993) [arXiv:hepth/9303123].

[4] M. Reuter, "Nonperturbative Evolution Equation for Quantum Gravity," Phys. Rev. D 57, 971 (1998) [arXiv:hep-th/9605030].

[5] W. Souma, "Non-trivial ultraviolet fixed point in quantum gravity," Prog. Theor. Phys. 102, 181 (1999) [arXiv:hep-th/9907027].

[6] O. Lauscher and M. Reuter, "Ultraviolet fixed point and generalized flow equation of quantum gravity," Phys. Rev. D 65, 025013 (2002) [arXiv:hep-th/0108040].

[7] O. Lauscher and M. Reuter, "Is quantum Einstein gravity nonperturbatively renormalizable?," Class. Quant. Grav. 19, 483 (2002) [arXiv:hep-th/0110021].

[8] D. F. Litim, "Fixed points of quantum gravity," Phys. Rev. Lett. 92, 201301 (2004) [arXiv:hepth/0312114]. 
[9] M. Niedermaier, "The asymptotic safety scenario in quantum gravity: An introduction," Class. Quant. Grav. 24, R171 (2007) [arXiv:gr-qc/0610018].

[10] A. Codello, R. Percacci and C. Rahmede, "Ultraviolet properties of f(R)-gravity," Int. J. Mod. Phys. A 23, 143 (2008) [arXiv:0705.1769 [hep-th]].

[11] A. Codello, R. Percacci and C. Rahmede, "Investigating the Ultraviolet Properties of Gravity with a Wilsonian Renormalization Group Equation," Annals Phys. 324, 414 (2009) [arXiv:0805.2909 [hep-th]].

[12] D. Benedetti, P. F. Machado and F. Saueressig, "Asymptotic safety in higher-derivative gravity," Mod. Phys. Lett. A 24, 2233 (2009) [arXiv:0901.2984 [hep-th]].

[13] S. Weinberg, "Living with Infinities," arXiv:0903.0568 [hep-th].

[14] S. Weinberg, "Effective Field Theory, Past and Future," arXiv:0908.1964 [hep-th].

[15] S. Weinberg, "Asymptotically Safe Inflation," arXiv:0911.3165 [hep-th].

[16] K. S. Stelle, "Renormalization Of Higher Derivative Quantum Gravity," Phys. Rev. D 16, 953 (1977).

[17] K. S. Stelle, "Classical Gravity With Higher Derivatives," Gen. Rel. Grav. 9, 353 (1978).

[18] S. W. Hawking and T. Hertog, "Living with Ghosts," Phys. Rev. D 65, 103515 (2002) [arXiv:hep-th/0107088].

[19] T. Clunan and M. Sasaki, "Tensor ghosts in the inflationary cosmology," arXiv:0907.3868 [hep-th].

[20] J. Julve and M. Tonin, "Quantum Gravity With Higher Derivative Terms," Nuovo Cim. B 46, 137 (1978).

[21] A. Salam and J. A. Strathdee, "Remarks On High-Energy Stability And Renormalizability Of Gravity Theory," Phys. Rev. D 18, 4480 (1978).

[22] R. Floreanini and R. Percacci, "The Renormalization group flow of the Dilaton potential," Phys. Rev. D 52, 896 (1995) [arXiv:hep-th/9412181].

[23] D. Benedetti, P. F. Machado and F. Saueressig, "Four-derivative interactions in asymptotically safe gravity," arXiv:0909.3265 [hep-th].

[24] A. Bonanno and M. Reuter, "Renormalization group improved black hole spacetimes," Phys. Rev. D 62, 043008 (2000) [arXiv:hep-th/0002196].

[25] A. Bonanno and M. Reuter, "Spacetime structure of an evaporating black hole in quantum gravity," Phys. Rev. D 73, 083005 (2006) [arXiv:hep-th/0602159]. 
[26] A. Bonanno, "Astrophysical implications of the Asymptotic Safety Scenario in Quantum Gravity," arXiv:0911.2727 [hep-th].

[27] K. Falls, D. F. Litim and A. Raghuraman, "Black holes and asymptotically safe gravity," arXiv:1002.0260 [hep-th].

[28] S. W. Hawking, "Particle Creation By Black Holes," Commun. Math. Phys. 43, 199 (1975) [Erratum-ibid. 46, 206 (1976)].

[29] K. Schwarzschild, "On the gravitational field of a sphere of incompressible fluid according to Einstein's theory," Sitzungsber. Preuss. Akad. Wiss. Berlin (Math. Phys. ) 1916, 424 (1916) [arXiv:physics/9912033].

[30] K. Schwarzschild, "On The Gravitational Field Of A Mass Point According To Einstein's Theory," Sitzungsber. Preuss. Akad. Wiss. Berlin (Math. Phys. ) 1916, 189 (1916) [arXiv:physics/9905030].

[31] D. F. Litim, "Optimisation of the exact renormalisation group," Phys. Lett. B 486, 92 (2000) [arXiv:hep-th/0005245].

[32] D. F. Litim, "Optimised renormalisation group flows," Phys. Rev. D 64, 105007 (2001) [arXiv:hep-th/0103195].

[33] I. G. Avramidi and A. O. Barvinsky, "Asymptotic Freedom In Higher Derivative Quantum Gravity," Phys. Lett. B 159, 269 (1985).

[34] G. de Berredo-Peixoto and I. L. Shapiro, "Higher derivative quantum gravity with GaussBonnet term," Phys. Rev. D 71, 064005 (2005) [arXiv:hep-th/0412249].

[35] A. Codello and R. Percacci, "Fixed Points of Higher Derivative Gravity," Phys. Rev. Lett. 97, 221301 (2006) [arXiv:hep-th/0607128].

[36] G. W. Gibbons and S. W. Hawking, "Action Integrals And Partition Functions In Quantum Gravity," Phys. Rev. D 15, 2752 (1977).

[37] K. Srinivasan and T. Padmanabhan, "Particle production and complex path analysis," Phys. Rev. D 60, 024007 (1999) [arXiv:gr-qc/9812028].

[38] M. K. Parikh and F. Wilczek, "Hawking radiation as tunneling," Phys. Rev. Lett. 85, 5042 (2000) [arXiv:hep-th/9907001].

[39] D. A. Easson, "Hawking radiation of nonsingular black holes in two dimensions," JHEP 0302, 037 (2003) [arXiv:hep-th/0210016].

[40] M. Trodden, V. F. Mukhanov and R. H. Brandenberger, "A Nonsingular two-dimensional 
black hole," Phys. Lett. B 316, 483 (1993) [arXiv:hep-th/9305111].

[41] V. F. Mukhanov and R. H. Brandenberger, "A Nonsingular universe," Phys. Rev. Lett. 68, 1969 (1992). 


\section{Appendix: Curvature invariants}

The curvature invariants appearing in the action (1) evaluated in terms of the line element (9):

$$
\begin{aligned}
& R=\frac{4 A^{2}-4 A^{2} B-4 r A B A^{\prime}+r^{2} B A^{\prime 2}-4 r A^{2} B^{\prime}-r^{2} A A^{\prime} B^{\prime}-2 r^{2} A B A^{\prime \prime}}{2 r^{2} A^{2}} \\
& R^{2}=\frac{1}{4 r^{4} A^{4}}\left(-\left(r^{2} B A^{\prime 2}\right)+r A\left(2 r B A^{\prime \prime}+A^{\prime}\left(r B^{\prime}+4 B\right)\right)\right. \\
& \left.+4 A^{2}\left(r B^{\prime}+B-1\right)\right)^{2} \\
& R_{\mu \nu} R^{\mu \nu}=\frac{1}{8 r^{4} A^{4}}\left(r^{4} B^{2} A^{\prime 4}+4 A^{4}\left(4(-1+B)^{2}+4 r(-1+B) B^{\prime}+3 r^{2} B^{\prime 2}\right)\right. \\
& +4 r A^{3}\left(A^{\prime}\left(4(-1+B) B+2 r B B^{\prime}+r^{2} B^{\prime 2}\right)+2 r^{2} B B^{\prime} A^{\prime \prime}\right) \\
& +r^{2} A^{2}\left(A^{\prime 2}\left(12 B^{2}+r^{2} B^{\prime 2}\right)+4 r B A^{\prime}\left(2 B+r B^{\prime}\right) A^{\prime \prime}+4 r^{2} B^{2} A^{\prime \prime 2}\right) \\
& \text { - } \left.2 r^{3} A B A^{\prime 2}\left(r A^{\prime} B^{\prime}+2 B\left(A^{\prime}+r A^{\prime \prime}\right)\right)\right) \\
& R_{\mu \nu \lambda \sigma} R^{\mu \nu \lambda \sigma}=\frac{1}{4 r^{4} A^{4}}\left(r^{4} B^{2} A^{\prime 4}+8 A^{4}\left(2(-1+B)^{2}+r^{2} B^{\prime 2}\right)\right. \\
& +-2 r^{4} A B A^{\prime 2}\left(A^{\prime} B^{\prime}+2 B A^{\prime \prime}\right)+A^{2}\left(r^{2} A^{\prime 2}\left(8 B^{2}+r^{2} B^{\prime 2}\right)\right. \\
& \left.\left.+4 r^{4} B A^{\prime} B^{\prime} A^{\prime \prime}+4 r^{4} B^{2} A^{\prime \prime 2}\right)\right)
\end{aligned}
$$

